\title{
A 1.4 GHz radio continuum and polarization survey at medium Galactic latitudes
}

\section{Observation and reduction technique}

\author{
B. Uyanıker, E. Fürst, W. Reich, P. Reich, and R. Wielebinski \\ Max-Planck-Institut für Radioastronomie, Postfach 2024, 53010 Bonn, Germany
}

Received December 4, 1997; accepted April 27, 1998

\begin{abstract}
A radio continuum survey at medium Galactic latitudes with the Effelsberg 100-m telescope is being carried out at a centre frequency of $1.4 \mathrm{GHz}$ in total power and linear polarization. Areas up to $\pm 20^{\circ}$ of Galactic latitude are now being observed at a sensitivity of $15 \mathrm{mK} T_{\mathrm{B}}$ in total intensity and $8 \mathrm{mK} T_{\mathrm{B}}$ in linear polarization with an angular resolution of 9'35. This paper describes the observing and reduction technique applied which results in absolutely calibrated maps. The methods are illustrated by examples of images from the survey.
\end{abstract}

Key words: polarization — methods: data analysis - techniques: polarimetric - surveys galaxy: structure — radio continuum: ISM

\section{Introduction}

The interaction between the Galactic magnetic field and interstellar clouds leads to a variety of radio emitting structures, shells, filaments, and loops. Many of these features are linearly polarized. They extend up to high Galactic latitudes but they are very faint, an order of magnitude fainter than the large-scale diffuse Galactic emission. However, the study of these structures may provide important clues for the understanding of the "disk-halo connection". The interpretation favours chimneys, fountains, Parker loops or superbubbles created by OB associations to transfer material from the disk into the halo. They will deform and compress the Galactic magnetic field. If these structures account for the rather smooth nonthermal background, they have to be sufficiently numerous and large.

The Galactic region above $b=+4^{\circ}$ and below $b=-4^{\circ}$, where the just mentioned structures play an important

Send offprint requests to: W. Reich role, has never been studied in a systematic way. Largescale surveys covering this area either suffer from low angular resolution or they miss the diffuse emission as it is the case for the 1.4 GHz VLA-survey (Condon et al. 1996). A combination of $1.4 \mathrm{GHz}$ data from the Effelsberg telescope with high angular resolution data from the VLA is of particular importance to separate compact background sources from faint extended Galactic structures. An example has already been shown and discussed by Fürst et al. (1998).

One of the milestones in the research of Galactic magnetic fields is due to Brouw \& Spoelstra (1976) and Spoelstra (1984). They have surveyed the northern sky in linear polarization with the Dwingeloo 25-m telescope at $1.411 \mathrm{GHz}$ at an angular resolution of about half a degree. They also compiled maps of the linear polarization at frequencies between $408 \mathrm{MHz}$ and $1.411 \mathrm{GHz}$. At $2.7 \mathrm{GHz}$ Junkes et al. (1987) published a survey of the linear polarization of the Galactic plane $\left(|b| \leq 1.5\right.$ and $\left.4.9 \leq \ell \leq 76^{\circ}\right)$. Recently, the southern Galactic plane $\left(|b| \leq 5^{\circ}\right)$ was surveyed at $2.4 \mathrm{GHz}$ by Duncan et al. (1995). Aside from the Dwingeloo data (see the discussion in Sect. 5), all these polarization surveys are constrained to the Galactic plane. On the low frequency side, at $327 \mathrm{MHz}$, Wieringa et al. (1993) observed small-scale variations in polarization at high latitudes with the Westerbork synthesis telescope.

The knowledge of the magnetic field in the Galactic halo even in the immediate vicinity of the Galactic plane is limited, because of the lack of observations of the linear polarization at the proper angular resolution and sensitivity. To fill this gap new observations at $1.4 \mathrm{GHz}$ are being carried out to cover the entire Galactic plane at medium Galactic latitudes $\left(|b| \leq 20^{\circ}\right)$ visible at Effelsberg. This survey will reach a sensitivity at total intensity close to the confusion limit $\left(\sim 15 \mathrm{mK} T_{\mathrm{B}}\right)$ and an even higher sensitivity $\left(\sim 8 \mathrm{mK} T_{\mathrm{B}}\right)$ at linear polarization.

We discuss the general survey parameters and observing method in Sect. 2. An absolute calibration method 
for the total intensity data is described in Sect. 3. The method developed to overcome the usual problem of polarimetric observations, the instrumental polarization, is demonstrated in Sect. 4 . In Sect. 5 we propose a procedure to adjust the polarization data to an absolute temperature scale.

\section{Observing method and data reduction}

The observations of the survey are carried out with the two-channel $1.3-1.7 \mathrm{GHz}$ receiver installed in the primary focus of the Effelsberg 100-m telescope. The receiver is equipped with cooled HEMT amplifiers recording the left- and right-hand circularly polarized component simultaneously (see Schmidt \& Zinz 1994). These amplifiers are of very high stability, and total intensity observations are limited by confusion which is reached after about $2 \mathrm{~s}$ of integration time. An IF-polarimeter converts the circularly polarized component into linear components (Stokes parameter $U$ and $Q$ ). Stokes parameter $I$ is obtained by simply adding both circularly polarized components.

The method of observation is to scan each field along constant Galactic latitude as well as along constant Galactic longitude. Each field was measured at least twice. The telescope parameters relevant to the $1.4 \mathrm{GHz}$ survey are listed in Table 1.

Table 1. Parameters for the survey

\begin{tabular}{lr}
\hline Parameter & Value \\
\hline Centre Frequency & $1.4 \mathrm{GHz}^{*}$ \\
Bandwidth & $20 \mathrm{MHz}^{*}$ \\
Integration time per point & $2 \mathrm{~s}$ \\
System temperature & $26 \mathrm{~K}$ \\
Typical rms noise in total power & $15 \mathrm{mK}$ \\
Typical rms noise in polarization & $8 \mathrm{mK}$ \\
$T_{\mathrm{B}} / S$ & $2.12 \pm 0.02 \mathrm{~K} / \mathrm{Jy}$ \\
Telescope beamwidth & $9^{\prime} .35 \pm 00^{\prime} .04$ \\
Typical scan length & $10^{\circ}$ \\
Scan interval & $4^{\prime}$ \\
Scanning velocity & $4^{\prime} / \mathrm{s}$ \\
\hline
\end{tabular}

* Sometimes interference makes a shift of the centre frequency and/or a reduction of the bandwidth necessary.

Daytime observations cause inacceptable distortions in both the total intensity and the polarization data due to contribution of solar emission to the far sidelobes of the telescope (Kalberla et al. 1980). Therefore all observations are carried out at nighttime, when additional ionospheric Faraday rotation effects are minimal and included by corrections inferred by observations of calibration sources. Calibration sources were 3C 286, 3C 138 and 3C 48, where $3 \mathrm{C} 286$ served as the primary calibrator both in total intensity and polarization. The scale accuracy for total and

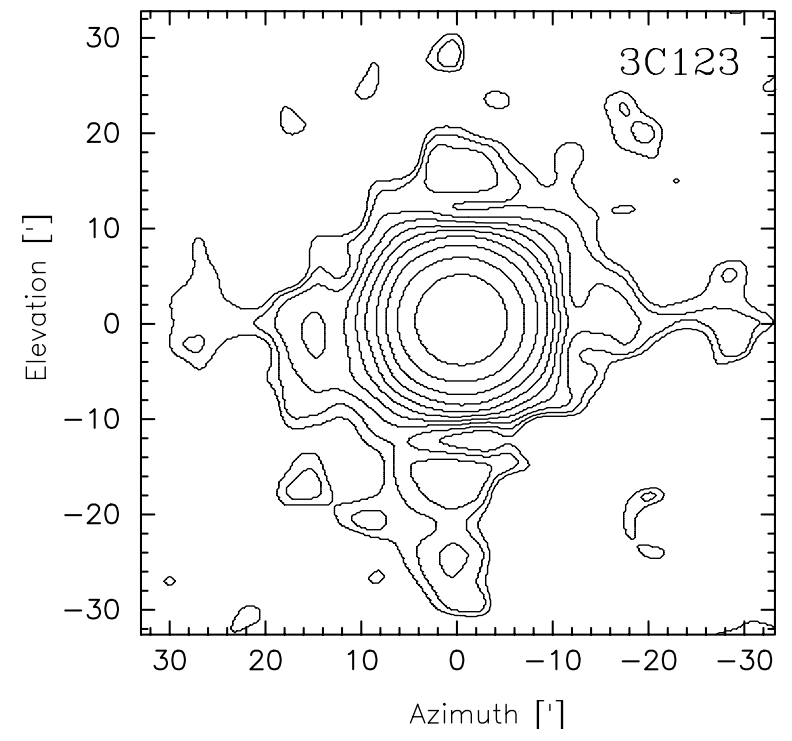

Fig. 1. $1.4 \mathrm{GHz}$ beam map covering an area of $1.1 \times 1.1$. Contours run in steps of $3 \mathrm{~dB}$. The lowest contour is at $-30 \mathrm{~dB}$ and the highest contour is at $-3 \mathrm{~dB}$ of the maximum

polarized intensities is better than $5 \%$. As seen from their coordinates, calibration sources have large angular separations on the sky, so that at least one of them is accessible at all times. Their polarization properties are listed in Table 2 and are taken from the list of Tabara \& Inoue (1980).

Table 2. Major calibration sources

\begin{tabular}{lrrccl}
\hline Source & \multicolumn{1}{c}{$\ell$} & $b$ & $S_{21 \mathrm{~cm}} / \mathrm{Jy}$ & $\%$ Pol. & PA \\
\hline 3C 286 & 56.5 & +80.7 & 14.4 & 9.3 & 32 \\
3C 138 & 187.4 & -11.3 & 9.5 & 7.9 & 169.9 \\
3C 48 & 134.0 & -28.7 & 15.9 & 0.4 & 139.7 \\
\hline
\end{tabular}

In Fig. 1 we show a beam map at $1.4 \mathrm{GHz}$ observed in the $\mathrm{Az} / \mathrm{El}$-system on $3 \mathrm{C} 123$, which at that frequency shows no measurable variation with elevation. Compact background sources in the field of $3 \mathrm{C} 123$ have been removed. The dynamic range exceeds $30 \mathrm{~dB}$. The maximum of the first sidelobes, which are enhanced by the four subreflector support legs, are at a level of about $-20 \mathrm{~dB}$. The cross-polarization lobes of Stokes parameters $U$ and $Q$ are dominated by a main beam component, which varies with parallactic angle. A correction of that instrumental response is described below.

The first stage of the data reduction made use of the standard "Toolbox" procedure (CONT2, von Kap-herr 1977) for continuum and polarization observations with the Effelsberg telescope. From the tabulated scans calibrated maps were computed for all the four data channels (two times $I, U$ and $Q$ ) recorded. A linear baseline using data points at the end of each scan was subtracted 
from each channel. All later stages of the data reduction are based on the NOD2 program package (Haslam 1974). Spiky data were removed. Scanning effects were suppressed by using the method of unsharp masking developed by Sofue \& Reich (1979). Finally, the two maps observed in orthogonal directions for each of the Stokes parameters have been added using the PLAIT program (Emerson \& Gräve 1988), which reduces "scanning effects" from individual distortions or by the baseline setting procedure in the case of an emission structure located at the edge of a map by appropriate weighted addition of the Fourier transforms of both maps. The polarization $U$ and $Q$ maps were corrected for instrumental polarization as described in Sect. 4 before combining the maps observed in orthogonal directions.

\section{Absolute calibration of the Effelsberg total intensity maps}

From the observation and the method of data reduction it is obvious that neither the total intensity nor the polarization maps are on an absolute temperature scale. The total intensity maps are calibrated to an absolute scale using the 1.4 GHz northern sky survey by Reich (1982) and Reich \& Reich (1986) carried out with the Stockert 25-m telescope. This procedure has already been described by Reich et al. (1990) when calibrating the Effelsberg 1.4 GHz Galactic plane survey. The Stockert survey has a scale accuracy of $5 \%$ and an uncertainty of the absolute zero-level of $0.5 \mathrm{~K}$.

Briefly, the Effelsberg data and the Stockert data are convolved to an angular resolution slightly exceeding that of the Stockert $1.4 \mathrm{GHz}$ survey (HPBW 36') and the difference between the two data sets is added to the Effelsberg data. This procedure also improves the original Effelsberg maps since large-scale distortions by atmospheric and ground radiation variations are removed.

However, still existing faint baseline effects in the low resolution Stockert data are also added to the higher resolution Effelsberg data. Such distortions were found to exist on small scales but not on the large-scale background component, which is the missing component of the Effelsberg maps. A modified version to calibrate the Effelsberg maps absolutely was introduced:

- Convolve the Effelsberg map to the resolution of the Stockert map $\left(36^{\prime}\right)$. Decompose this map into a largescale component (Eff.back) and a map with small-scale structures using the "background filtering method" (Sofue \& Reich 1979) with a $3^{\circ}$ Gaussian beam for smoothing.

- Subtract the $2.8 \mathrm{~K}$ isotropic background component from the Stockert data and convert the temperature scale from full beam into main beam $\left(T_{\mathrm{MB}} / T_{\mathrm{FB}}=\right.$ $1.55)$.

- Decompose the Stockert data to obtain the corresponding large-scale component (Sto.back) by using the same filtering parameters as for the Effelsberg data.

- Adjust Eff.back to Sto.back as described above, namely, add the difference of both large-scale component maps to the Effelsberg large-scale data and add the $2.8 \mathrm{~K}$ isotropic background component. The resulting map is ES.back.

- The final Effelsberg map (Eff.final) is given by:

$$
\text { Eff.final }=\text { Eff.orig }+ \text { ES.back }- \text { Eff.back. }
$$

We use the modified method only when there are nonnegligible scanning effects in the source component of the Stockert data. A sample region (towards $\ell \sim 50^{\circ}$ ) is given in Fig. 2 to illustrate the effect of the absolute calibration.

\section{Instrumental polarization}

One major goal of the new $1.4 \mathrm{GHz}$ survey is the sensitive mapping of polarized emission out of the Galactic plane. However, a problem is given by the relatively high instrumental polarization mainly introduced by the cooled broad-band polarization transducer and hybrid of the L-band Effelsberg receiver. A change of the bandwidth and centre frequency has a significant effect on the instrumental $U$ and $Q$ components. Other instrumental effects due to the antenna and feed characteristics, residual ellipticity of the polarimeter response and variations with time add, but are too small to be separated from the main effect.

An attempt was made to minimize these instrumental effects to a residual effect of the order of $1 \%$. We assume that the instrumental components $U_{\text {inst }}$ and $Q_{\text {inst }}$ scale with the total intensity $I$. When observing in an astronomical coordinate system the instrumental components depend on the parallactic angle $\phi$. A procedure was developed to correct for this effect. From the observations of the polarized calibration sources $3 \mathrm{C} 286$ and $3 \mathrm{C} 138$ at different parallactic angles, correction factors $f_{U}$ and $f_{Q}$ are calculated in such way that the nominal percentage polarization and the polarization angle are obtained.

The algorithm of the elimination of the instrumental effects is as follows: Suppose $U_{\text {act }}$ and $Q_{\text {act }}$ are the intrinsic values of a source in an astronomical coordinate system. $U_{\text {obs }}$ and $Q_{\text {obs }}$ are the observed values depending on the parallactic angle $\phi$. The instrumental values $U_{\text {inst }}$ and $Q_{\text {inst }}$ are:

$U_{\text {inst }}(\phi)=U_{\text {obs }}(\phi)-U_{\text {act }}$

$Q_{\text {inst }}(\phi)=Q_{\text {obs }}(\phi)-Q_{\text {act }}$.

The instrumental polarization angle $P A_{\text {inst }}$ and polarization intensity $P I_{\text {inst }}$ are:

$P A_{\text {inst }}(\phi)=\frac{1}{2} \tan ^{-1}\left[\frac{U_{\text {inst }}(\phi)}{Q_{\text {inst }}(\phi)}\right]$
$P I_{\text {inst }}(\phi)=\sqrt{U_{\text {inst }}^{2}(\phi)+Q_{\text {inst }}^{2}(\phi)}$. 


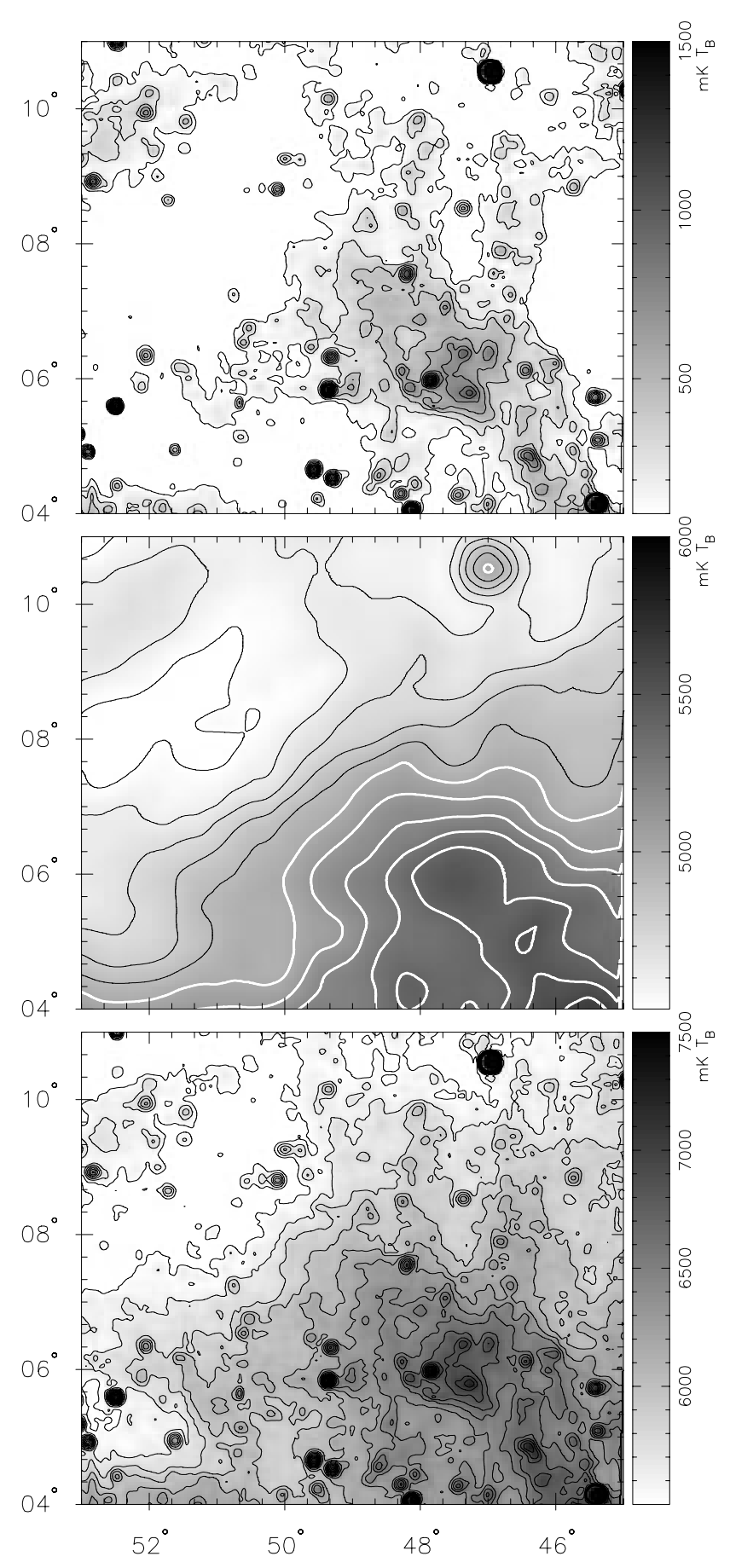

Fig. 2. A small section from the survey, towards $\ell \sim 50^{\circ}$, illustrates the calibration of the total intensity data to the absolute temperature scale. The panels, from top to bottom, display the Effelsberg (contours start at $100 \mathrm{mK}$ and are plotted in $150 \mathrm{mK}$ steps) and Stockert $1.4 \mathrm{GHz}$ (contours start at $4500 \mathrm{mK}$ in $100 \mathrm{mK}$ steps, full beam brightness scale) measurements and the combination of the two, respectively. Contours for the combined map (bottom) run from $5500 \mathrm{mK}$ in steps of $150 \mathrm{mK}$

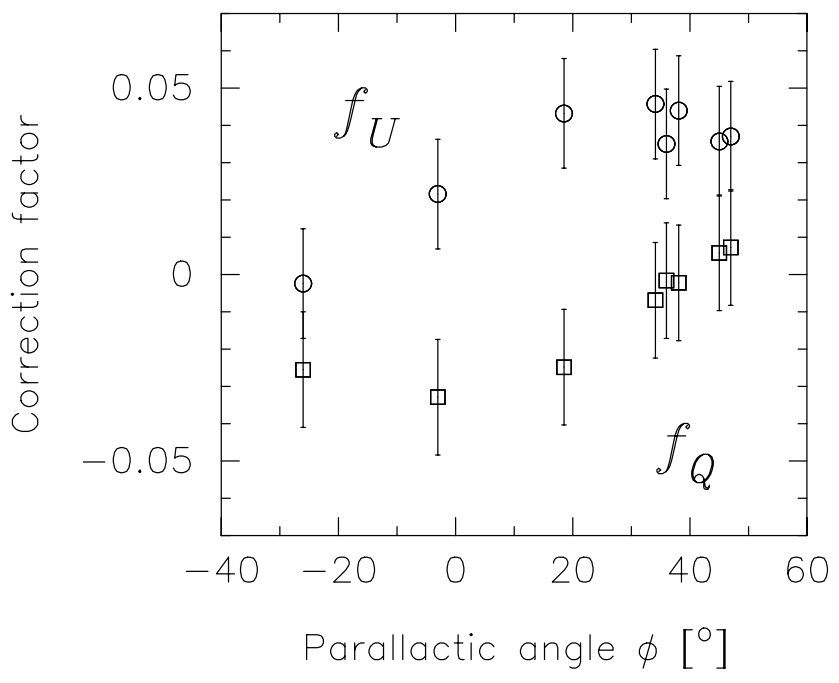

Fig. 3. Variation of the calculated $f_{U}$ and $f_{Q}$ factors with respect to the parallactic angle for 3C 286 during one observing session. Error bars are the standard deviations of the factors

The correction factors $f_{U}$ and $f_{Q}$ are then:

$$
\begin{gathered}
f_{U}(\phi)=\frac{P I_{\text {inst }}(\phi)}{I_{\text {obs }}} \sin \left[2\left(P A_{\text {inst }}(\phi)-\phi\right)\right] \\
f_{Q}(\phi)=\frac{P I_{\text {inst }}(\phi)}{I_{\text {obs }}} \cos \left[2\left(P A_{\text {inst }}(\phi)-\phi\right)\right] .
\end{gathered}
$$

In Fig. 3 we show, as an example, the data for $f_{U}$ and $f_{Q}$ from observations of $3 \mathrm{C} 286 . f_{U}$ and $f_{Q}$ are determined for each session. It is obvious that $f_{U}$ and $f_{Q}$ vary with parallactic angle, which needs to be taken into account when correcting a survey map. According to the introduced procedure each pixel $I$ of a map is multiplied with the appropriate $f_{U}$ and $f_{Q}$. The resulting $U$ and $Q$ components of the instrumental polarization are transformed into parallactic angle corrected components for each pixel of an $U$ and $Q$ map and subtracted from the observed $U$ and $Q$. The procedure corrects for most of the instrumental effects. The corrected $U$ and $Q$ maps are then used to calculate the polarized intensity $P I$ and the polarization angle $P A$.

It has been found that the correction effect on the large-scale polarization emission is not significant. Strong sources, however, cause distortions which are clearly minimized by the described procedure. In Fig. 4 we illustrate the effect of our correction procedure. We note that instrumental effects do not always act in a way to increase the observed polarization. Depending on the arrangement and variations in $U$ and $Q$ it is equally possible to observe the reverse of this effect.

\section{Procedure for the absolute polarization adjustment}

As was mentioned earlier the large-scale information in the polarization maps is lost due to the baseline fitting 


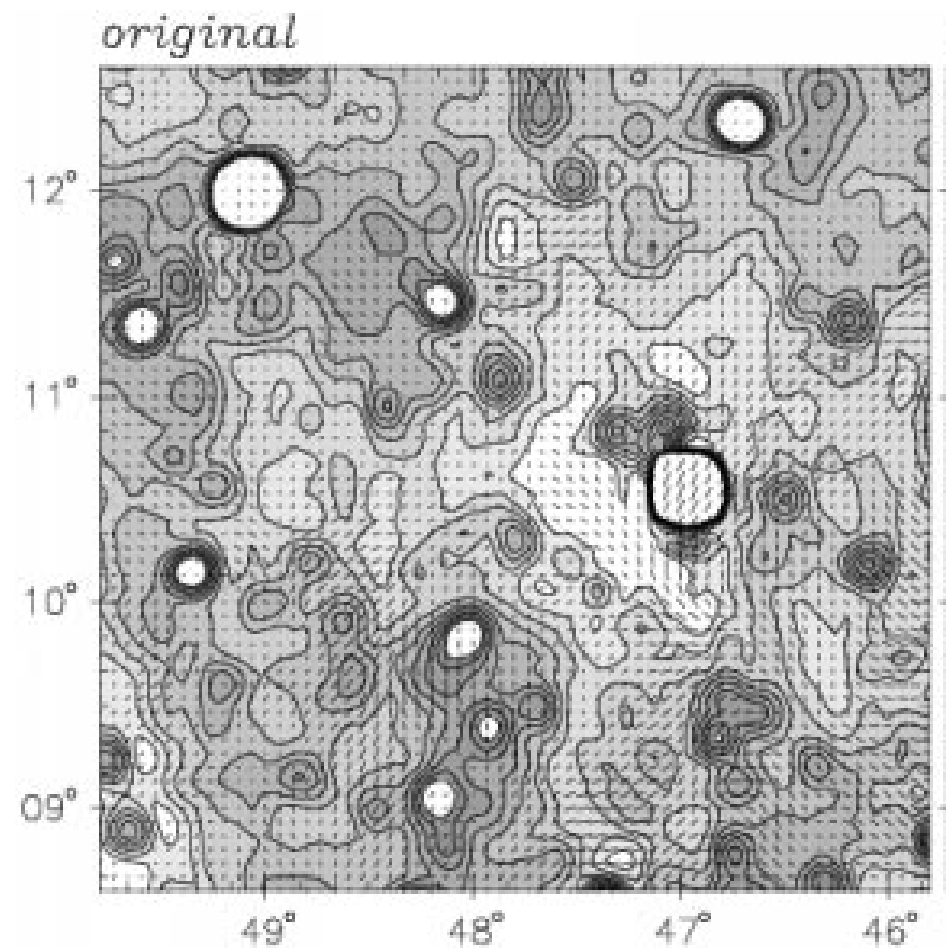

corrected

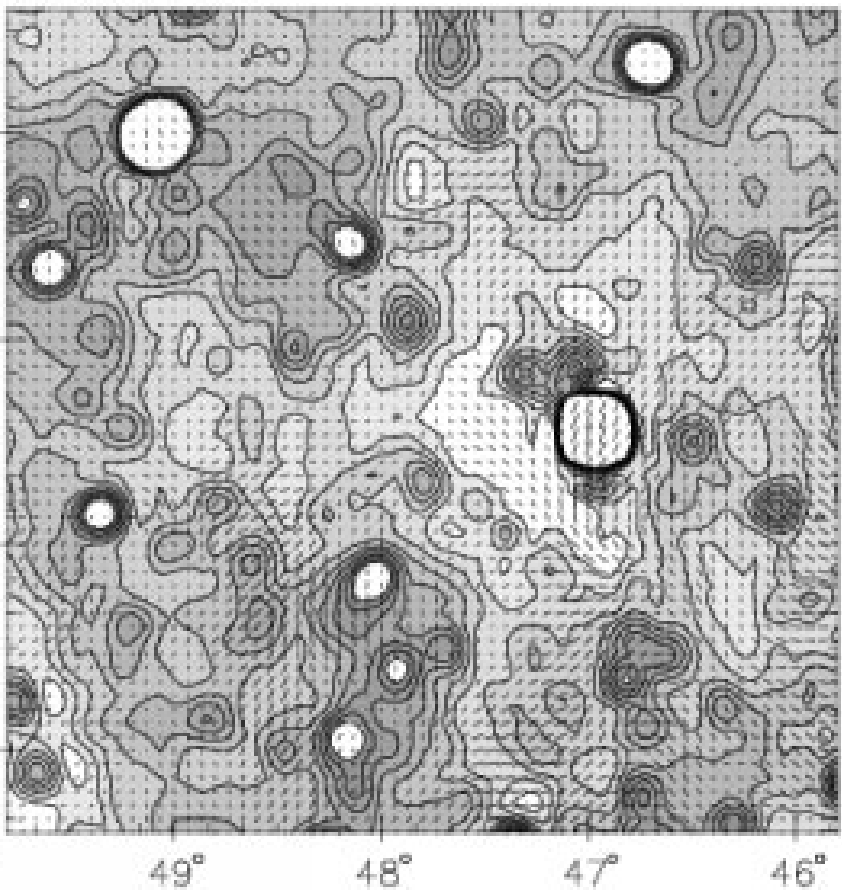

original

corrected
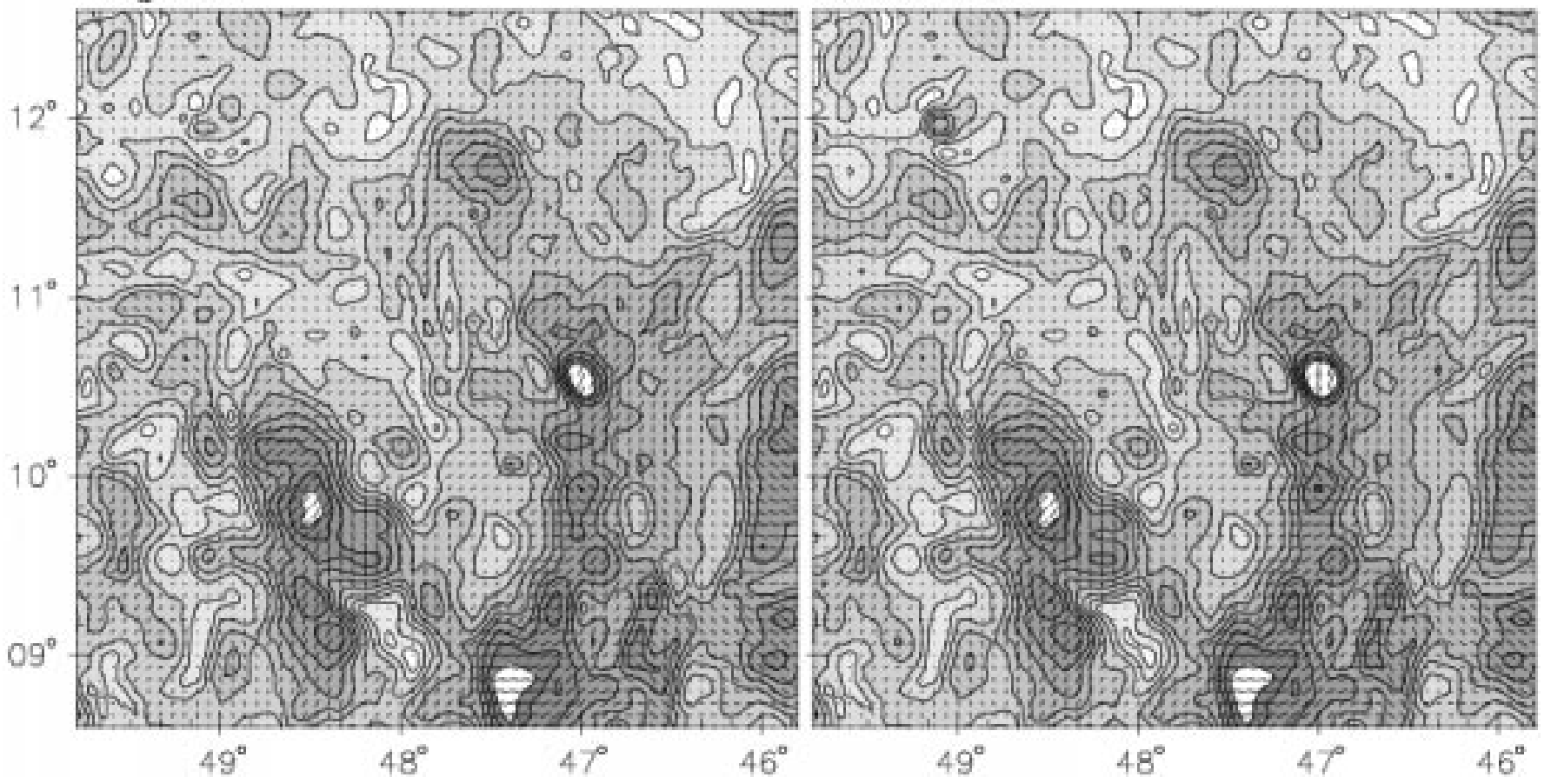

Fig. 4. A sample region to illustrate the effect of the procedure to eliminate the instrumental polarization. The upper panel displays the same region before (left) and after (right) the correction. Contours show the total intensity starting from $-50 \mathrm{mK}$ with $50 \mathrm{mK}$ intervals. The strong source around $\ell \sim 47^{\circ}$ is $3 \mathrm{C} 386$ with a flux density of $6.5 \mathrm{Jy}$. Its percentage polarization is $2.7 \%$ after the procedure is applied. In the lower panel the polarized intensity data of the same regions are displayed in grey scale. Contour levels run starting from $10 \mathrm{mK}$ with $30 \mathrm{mK}$ steps. Again the original data are at left and corrected data are at right. The plotted electric field vectors are scaled to the polarized intensity and astronomical coordinates such that $100 \mathrm{mK}$ correspond to a vector of length 1.5 
procedure. In addition, residual ground radiation effects may show up in view of the large sizes of the observed maps. As in the case of total power maps, the polarization emission needs to be adjusted to an absolute level. However, there exists no complete and regularly gridded low resolution polarization survey which is absolutely calibrated. Fortunately, a large set of linear polarization observations at $1.4 \mathrm{GHz}$ obtained with the Dwingeloo $25-\mathrm{m}$ telescope exists (Brouw \& Spoelstra 1976). These data are absolutely calibrated and corrected for all kinds of instrumental effects and therefore match the requirements for this task. However, the data are not on a regular grid and are significantly undersampled. This data set has to be regridded to be used to adjust our polarization measurements. The polarized intensity and polarization angle data from the Dwingeloo survey have been provided by Dr. Spoelstra in digitized form.

Using the $P I$ and $P A$ values from this measurements we calculated $U$ and $Q$ values and regridded the undersampled Dwingeloo data on the grid of the Effelsberg maps. However, in some regions data points are too separated $\left(2^{\circ}\right.$ or more) for an absolute calibration, and in other weakly polarized regions an $\mathrm{S} / \mathrm{N}$-ratio of 2 or less does not allow a proper adjustment.

We tried two methods to interpolate the Dwingeloo data on the same grid as the Effelsberg maps. One way to do this is an interpolation between the data points using a "cubic-spline interpolation". However, we found that a cubic-spline interpolation introduces distortions at the corners of the maps and data from a much larger area must be used to avoid this problem. Moreover, a single high intensity data point affects surrounding low intensity data up to a large distance. Data points must be weighted with respect to the distance. Hence, we found that a cubicspline interpolation is inadequate for most of our regions.

A successful method is to weigh data points by their distances to the required map element. We used the approach $\exp (-\alpha \mathcal{R})$ in which $\alpha$ is a constant. We calculate for each pixel of an Effelsberg map the Dwingeloo $U$ and $Q$ data within a radius, $\mathcal{R}$, contributing with a weight as given above. For our case we found a value of $6^{\circ}$ for $\mathcal{R}$ with $\alpha=1$ to be satisfactory.

The reconstructed $U, Q$ and $P I$ Dwingeloo maps of a test region at the $4^{\prime}$ grid of the Effelsberg maps are given in Fig. 5 .

Figure 6 shows the $U$ and $Q$ maps of the original Effelsberg measurements of an area, which is a small section of the Dwingeloo map shown in Fig. 5. In the higher resolution Effelsberg maps numerous small-scale polarization structures are visible, which are smoothed out by the large Dwingeloo beam.

The two data sets are combined as follows: The Effelsberg map is convolved to the Dwingeloo beam $\left(36^{\prime}\right)$ and subtracted from the Dwingeloo map. The difference is added to the original Effelsberg map. Figure 7 and Fig. 8 show the combination for $U$ and $Q$ and the correspond- ing $P I$ map. In these figures the small-scale structures are much less pronounced due to the addition of the strong large-scale polarized emission which varies in the range from $200 \mathrm{mK}$ to $800 \mathrm{mK}$ across the map (Fig. 5).

The absolutely calibrated $U$ and $Q$ maps may be decomposed into small-scale and large-scale features by standard methods for total intensity maps and relative $P I$ maps can be calculated. Some examples have been given by Uyanıker (1997).

Plots of the polarization vectors demonstrate the effect of adjusting the Effelsberg data to an absolute level. The polarization angle maps are presented as vector plots in Fig. 9. Numerous small-scale structures are visible in the original Effelsberg map. The polarization angle varies largely across the maps. However, the electric field vectors are almost constant in the combined Effelsberg-Dwingeloo map. The data of this map are the same as those for Fig. 8, but grey-scale representation is much more sensitive to small variations.

\section{Final remarks}

We described the observation and reduction technique for a sensitive $1.4 \mathrm{GHz}$ continuum and polarization survey with the Effelsberg 100-m telescope. We illustrated by an example the absolute calibration of the total intensity Effelsberg data by making use of the $1.4 \mathrm{GHz}$ Stockert survey and showed the effect on the Effelsberg polarization maps after adjusting the large-scale polarized emission with the Dwingeloo $1.4 \mathrm{GHz}$ measurements.

The methods used and introduced in this work have been found to be appropriate to conduct a $1.4 \mathrm{GHz}$ survey which is absolutely calibrated both in total and polarized intensity and corrected for instrumental polarization down to a level of $1 \%$.

Acknowledgements. We are very grateful to Dr. Titus Spoelstra for providing the Dwingeloo polarization data in digital form. We thank Dr. W.N. Brouw for helpful comments on the manuscript.

\section{References}

Brouw W.N., Spoelstra T.A.Th., 1976, A\&AS 26, 129

Condon J.J., Cotton W.D., Greisen E.W, Yin Q.F, Perley R.A., Broderick J.J., 1996, The NRAO VLA Sky Survey. In: NCSA Astronomy Digital Image Library, Associated Universities, Inc./National Radio Astronomy Observatory Duncan A.R, Stewart R.T, Haynes R.F., Jones K.L., 1995, MNRAS 277, 36

Emerson D.T., Gräve R., 1988, A\&A 190, 353

Fürst E., Reich W., Reich P., Uyanıker B., Wielebinski R., 1998. In: McLean B.J. et al. (eds.) Proc. IAU Symp. 179, New Horizons from Multi-Wavelength Sky Surveys. Kluwer, Dordrecht, p. 97

Haslam C.G.T., 1974, A\&AS 15, 333

Junkes N., Fürst E., Reich W., 1987, A\&AS 69, 451 

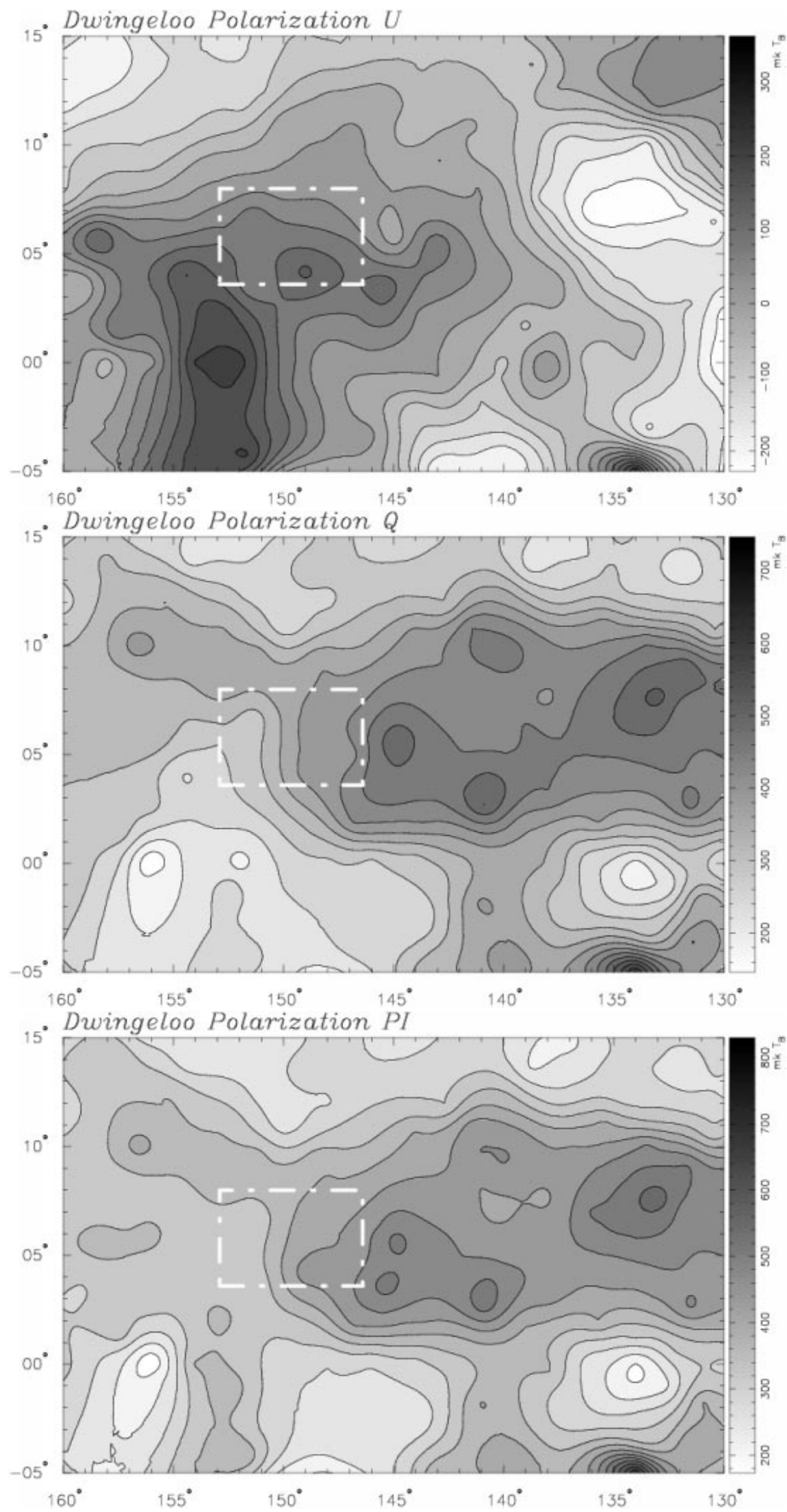

Fig. 5. Polarization maps reconstructed from the Dwingeloo data as explained in the text. The panels, from top to bottom, display the Stokes $U$ and $Q$ maps and polarization intensity, respectively. The area marked with dashed lines is the region observed with the Effelsberg telescope and used to demonstrate the method of absolute calibration for the polarization data 

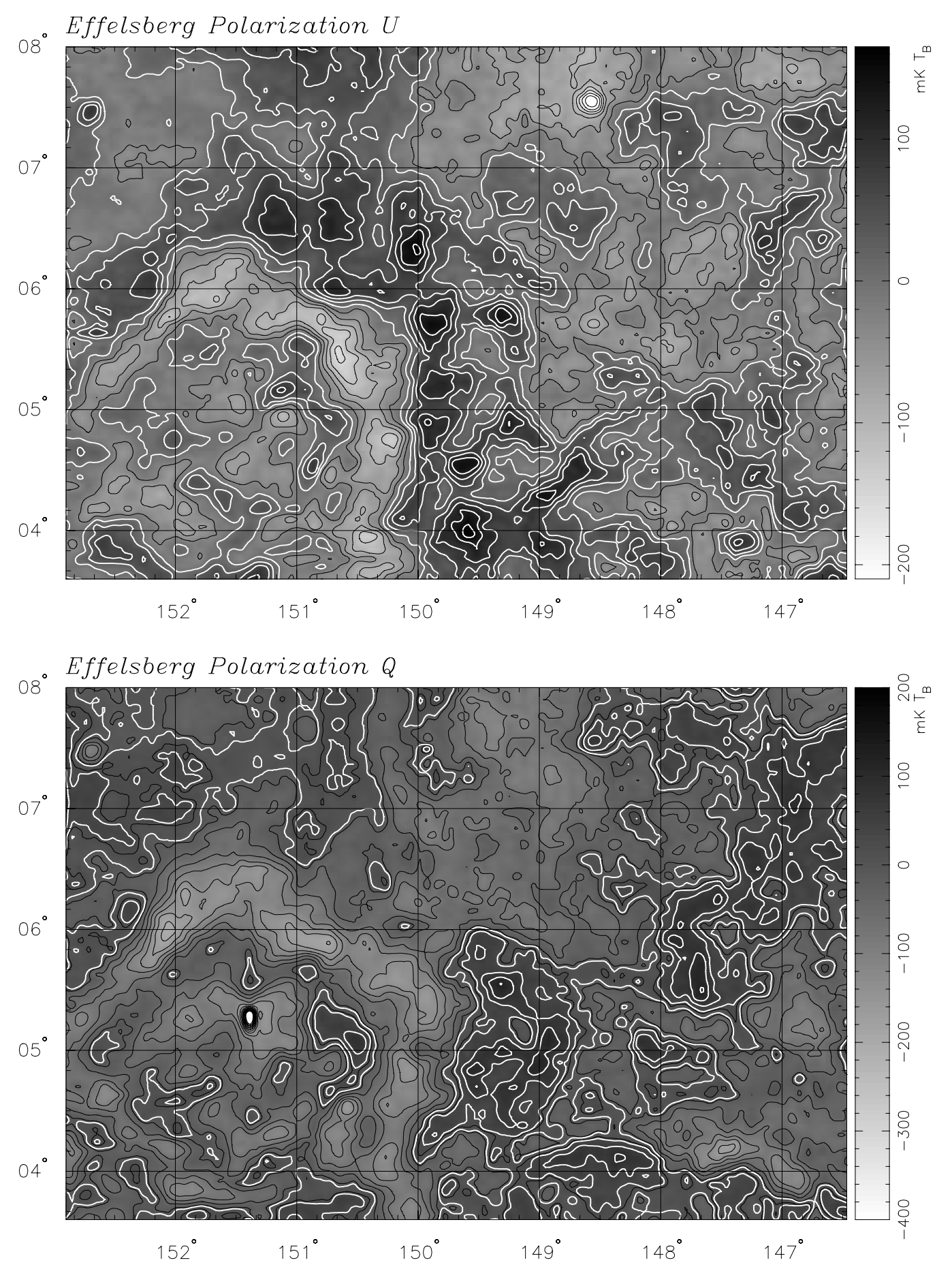

Fig. 6. Polarization $U$ and $Q$ maps from the Effelsberg $1.4 \mathrm{GHz}$ survey. Contours are plotted starting from the lowest value of the wedge and in steps of $30 \mathrm{mK} T_{\mathrm{B}}$. Contours starting from zero are plotted in white with the same steps

Kalberla P.M.W., Mebold U., Reich W., 1980, A\&A 82, 275

Reich P., Reich W., 1986, A\&AS 63, 205

Reich W., 1982, A\&AS 48, 219

Reich W., Reich P., Fürst E., 1990, A\&AS 83, 539

Schmidt A., Zinz W., 1994, Technischer Bericht Nr. 67-5, MPIfR Bonn, (update: http://www.mpifr-bonn.mpg.de /w3/technik/1_4ghz.html).

Sofue Y., Reich W., 1979, A\&AS 38, 251
Spoelstra T.A.Th., 1984, A\&A 135, 238

Tabara H., Inoue M., 1980, A\&AS 39, 379

Uyanıker B., 1997, PhD Thesis, Bonn University

von Kap-herr A., 1977, Technischer Bericht Nr. 40, MPIfR Bonn

Wieringa M.H., de Bruyn A.G., Jansen D., Brouw W.N., Katgert P., 1993, A\&A 268, 215 

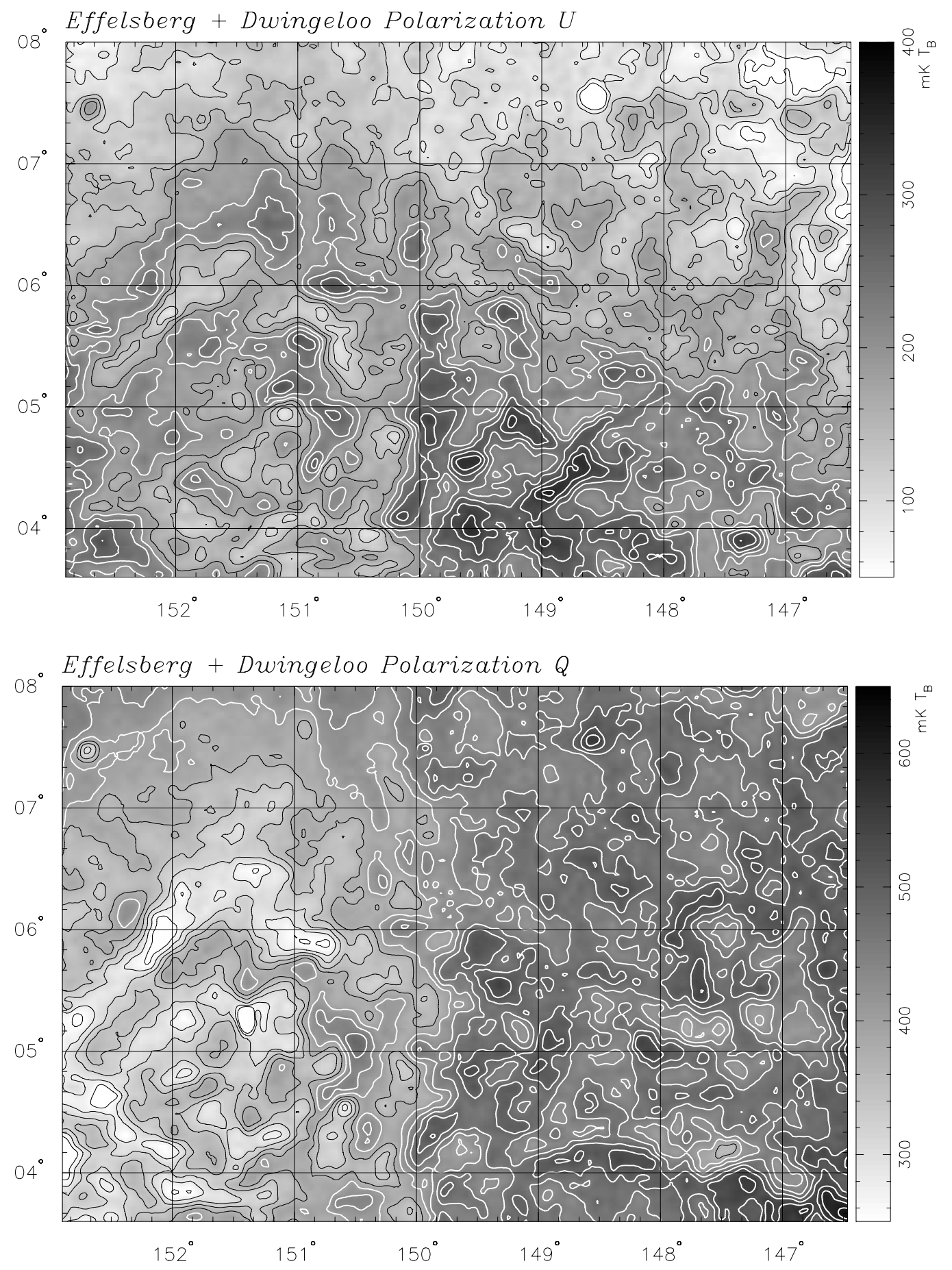

Fig. 7. Polarization $U$ and $Q$ maps after calibrating the Effelsberg polarization maps to absolute temperature scale. Contours are plotted starting from the lowest value of the wedge and in steps of $30 \mathrm{mK} T_{\mathrm{B}}$. For $U$ map the contours starting at $200 \mathrm{mK}$ $T_{\mathrm{B}}$ and for the $Q$ map the contours starting at $400 \mathrm{mK} T_{\mathrm{B}}$ are plotted in white. Contour steps are always $30 \mathrm{mK} T_{\mathrm{B}}$ 

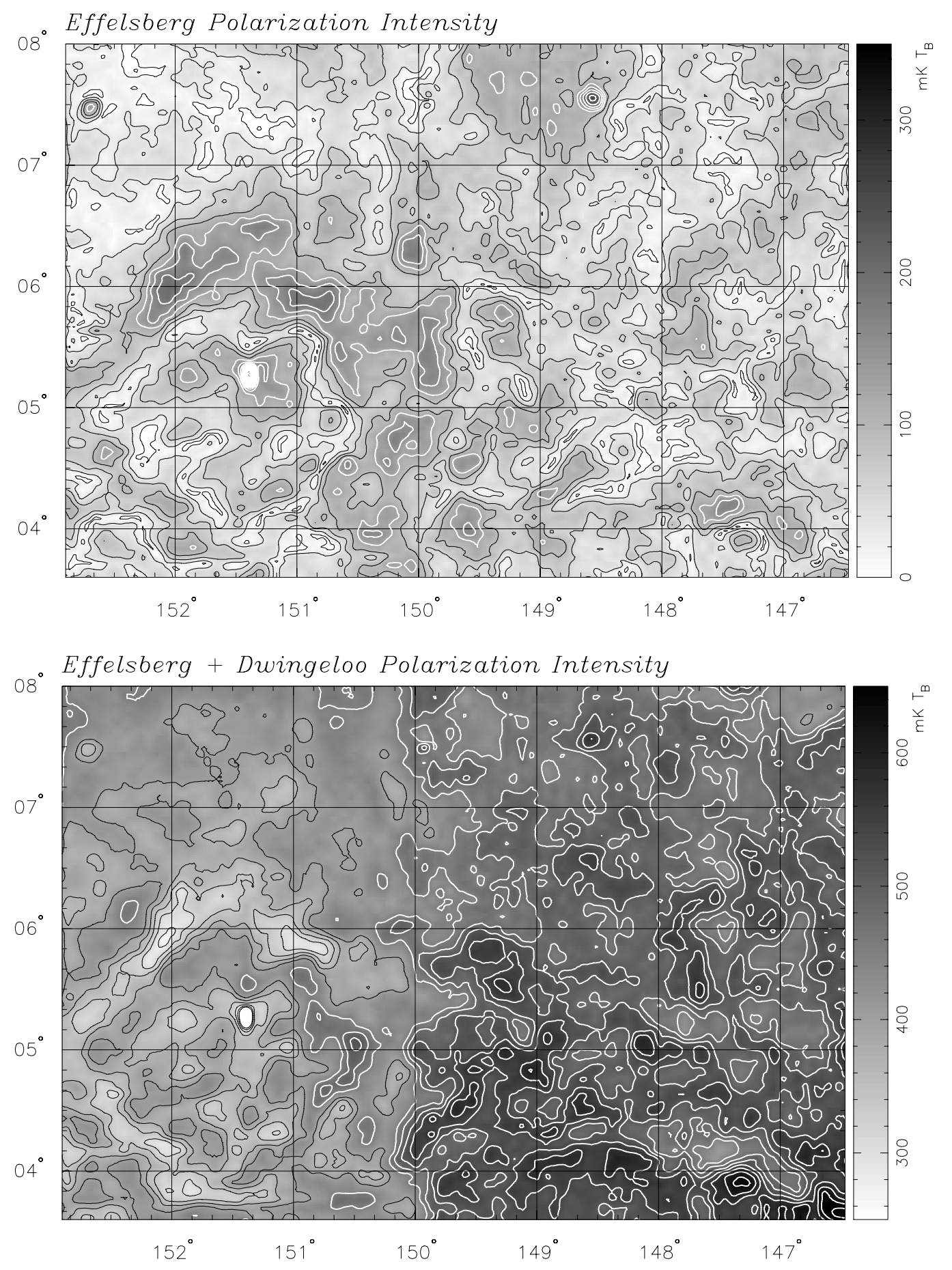

Fig. 8. Polarization intensity maps before (top) and after (bottom) calibrating the Effelsberg polarization maps to absolute temperature scale. Contours are plotted starting from the lowest value of the wedge and in steps of $30 \mathrm{mK} T_{\mathrm{B}}$. For the upper panel contours starting at $120 \mathrm{mK} T_{\mathrm{B}}$ and for the lower panel contours starting at $450 \mathrm{mK} T_{\mathrm{B}}$ are plotted in white 

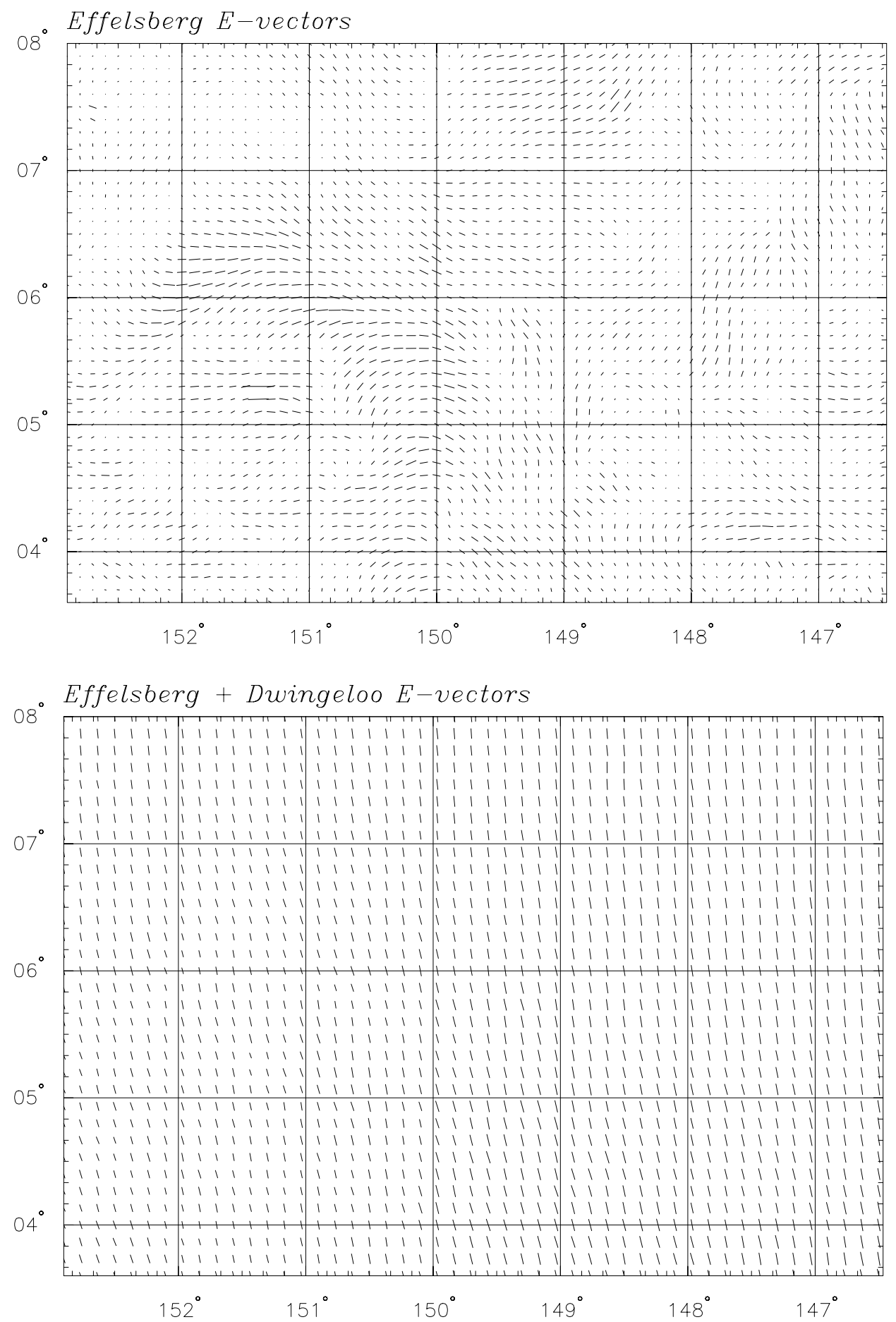

Fig. 9. Electric field vectors before (top) and after (bottom) calibrating the Effelsberg polarization maps to absolute temperature scale. The lower panel is essentially the same as Fig. 8. Due to the high polarization intensity of background polarization smallscale variations from the Effelsberg observations are hidden. Electric field vectors are scaled to the polarized intensity such that $100 \mathrm{mK}$ correspond to a vector of length $3^{\prime}$ for the upper panel and $1^{\prime}$ for the lower panel 